\title{
CARACTERIZACIÓN Y MODELAJE DE UN PARQUE ENERGÉTICO MARINO PARA EXPLOTAR LAS CORRIENTES OCEÁNICAS DEL CANAL DE LA MONA
}

Modeling and characterization of a marine current energy plant to exploit the Canal de la Mona oceanic currents

\author{
William Camilo Reynoso, Ph. D. ${ }^{1}$ \\ Francisco H. NúÑEZ RAmíreZ, MSc.
}

\begin{abstract}
Cómo citar: Camilo Reynoso, W., \& Núñez Ramírez, F. (2018). Caracterización y modelaje de un parque energético marino para explotar las corrientes oceánicas del Canal de la Mona. Ciencia, Ingenierías y Aplicaciones, 1(1), 47-60. doi:http://dx.doi. org/10.22206/cyap.2018.v1i1.pp47-60
\end{abstract}

\section{Resumen}

En esta nota técnica se considera el estudio y diseño de una superestructura energética capaz de producir energía mediante el aprovechamiento de las corrientes marinas del canal de la Mona. El objetivo de esta superestructura es explotar las corrientes como recurso natural sostenible para la República Dominicana con el fin de producir energía eléctrica. Además, se discutirá la posible implementación de un prototipo a escala que permita estimar y predecir el comportamiento de generadores de energía basados en el aprovechamiento de corrientes marinas. Para desarrollar el presente estudio se discutirá la posible implementación de un marco de trabajo basado en los siguientes pasos:

- $\quad$ Estudio del estado del arte actual, que incluirá los distintos tipos de turbinas, así como plataformas de fijación, líneas de transmisión de la energía y su transformación.

1 . Docente investigador de la Escuela de ingeniería, Decanato de ingeniería e informática, Universidad APEC, Avenida Máximo Gómez No. 72, El Vergel, Santo Domingo, República Dominicana. Correo electrónicowcamilor@gmail.com 
- Estudio del fondo marino del canal de la Mona, referente a la topología, la geología y las características climáticas.

- Desarrollo e implementación de sistemas de medición del flujo de corrientes. Este apartado incluye tanto el desarrollo como la ubicación y recopilación de información. Esto permitirá la generación de modelos y la estimación real del caudal.

- Análisis y fusión de datos. Selección del material y adecuación al entorno, tanto de las turbinas como de las plataformas de fijación marinas.

- Desarrollo de un prototipo para experimentación, predicción y adecuación de los resultados.

- $\quad$ Estudio de eficiencia y desarrollo de las infraestructuras de transmisión y conversión energéticas necesarias.

- Análisis de los resultados. Cuantificación económica y planificación en el tiempo, ambas necesarias para el desarrollo del proyecto.

Palabras clave: parque energético marino; turbinas acuáticas; energía renovable; caracterización estructural del enclave.

\begin{abstract}
This note considers the design of a marine current energy plant to exploit the energy generated by marine currents in the Canal de la Mona, near the Dominican Republic. The objective of this energy plant is to exploit marine currents as renewable energy resource for the Dominican Republic to generate electric energy. In addition, the article discusses the possible implementation of a small-scale prototype to estimate and predict the behavior of energy generators based on the use of marine currents in the considered environment.
\end{abstract}




\section{Introducción}

Uno de los grandes retos de la humanidad y, en consecuencia, de la República Dominicana tiene por objetivo auspiciar la transición hacia un sistema energético seguro, sostenible y competitivo. Por esta razón, en este artículo se busca estudiar la posibilidad del uso de las corrientes marinas para generar electricidad; dicha iniciativa puede contribuir significativamente a la reducción de la dependencia de los carburantes fósiles. Concretamente, cuando se analiza la realidad en la República Dominicana se enfatiza el hecho social de la carencia de energía eléctrica que soporta la población nacional. Así, pues, este país tiene un déficit promedio de potencia eléctrica de unos $300 \mathrm{MW}$ diarios y más de 300,000 hogares no tienen acceso a las redes de energía eléctrica.

\section{Caracterización del modelo para la plataforma marina}

Para la caracterización de un modelo de plataforma submarina para la generación eléctrica se requiere de un levantamiento completo y detallado del potencial energético de las corrientes marinas del litoral dominicano, que tiene un gran potencial al estar alimentado por las corrientes del Océano Atlántico, con su corriente del Golfo. Los investigadores estiman que esta corriente del Golfo transporta al norte unos 1,300 millones de Megavatios (MWatts), el equivalente a la producción de un millón de centrales energéticas; de estos, se espera explotar unos 1,400 Mwatts para alimentar la matriz energética dominicana a través de las corrientes que atraviesan el canal de la Mona, desde donde se estima obtener 12,096,000,000 KWhrs/ año, con unas 8,640 horas de extracción.

En la primera fase del proyecto se realizará el estudio de factibilidad para un modelo energético sostenible a través del aprovechamiento de las corrientes marinas del canal de la Mona. Se trata de un proyecto global que no solo incluye la generación de energía, sino también el 
transporte de esta a los puntos de consumo y el desarrollo de prototipos a escala. Los objetivos son los siguientes:

- Investigar el estado del arte actual referente al aprovechamiento de las corrientes marinas y su implementación en la República Dominicana.

- Desarrollar e implementar sistemas de medición del flujo de corrientes en el canal de la Mona. Generar y estimar los modelos de caudal.

- Estudiar las condiciones orográficas y climáticas del canal de la Mona.

- Recopilar datos. Seleccionar el material y adecuarlo al entorno, tanto de las turbinas como de las plataformas de fijación marinas.

- Desarrollar un prototipo a escala de bajo coste, a partir de los resultados obtenidos en las áreas.

- Estudiar la eficiencia y el desarrollo de las infraestructuras necesarias para la transmisión energética (red eléctrica marina-terrestre y estaciones transformadoras).

- Experimentar con el prototipo del sistema (plataforma, turbina).

- Analizar los resultados, las propuestas de mejora y el desarrollo futuro.

- Difundir los resultados (poderes públicos y compañías del sector).

El canal de la Mona, ubicado en la zona contigua de las aguas litorales dominicanas, es el lugar de comunicación natural entre el mar Caribe y el Océano Atlántico, limitado al este por el cabo Engaño y al oeste por la bahía de Mayagüez. Tiene una anchura variable de $107 \mathrm{~km}$. El eje del canal transcurre de este a oeste, con una distancia de $106 \mathrm{~km}$ sobre el paralelo $18^{\circ}$ Norte, $68^{\circ}$ Oeste. Este canal posee corrientes que pueden alcanzar las 3 millas náuticas/hora $(1.54 \mathrm{~m} / \mathrm{s})$ [1]-[3]. El potencial energético de las corrientes marinas del litoral dominicano es importante al estar alimentado por las corrientes del Océano Atlántico, con su corriente del Golfo. Los investigadores estiman que esta corriente transporta al norte unos 1,300 millones 
de megavatios, el equivalente a la producción de un millón de centrales energéticas. En los períodos de crecimiento de la marea se puede observar cómo la diferencia entre estas centrales provoca una gran entrada de agua superficial, llegando a generarse la formación de ondas superficiales que se desplazan dentro del mar Caribe. Las aguas territoriales de la República Dominicana (o, simplemente, mar dominicano), comprendidas entre sus costas, se extienden a una trazada de 6 millas náuticas (11 kilómetros) de aquellas. El Estado dominicano tiene facultades exclusivas de propiedad y jurisdicción sobre ellas, así como sobre su suelo y subsuelo. La plataforma continental o insular se entiende la parte de la línea costera sumergida, cuya profundidad está entre 200 metros y de 350 metros en el canal de la Mona. El límite exterior de la plataforma submarina forma lo que se llama zócalo continental insular. Dicha plataforma insular es reducida $\left(9,484 \mathrm{~km}^{2}\right)$; su anchura promedia unos $7.4 \mathrm{~km}$.

La Corriente Ecuatorial del sur se origina en el Golfo de Guinea, dirigiéndose también hacia el oeste $\mathrm{y}$, ante la interferencia del Cabo San Roque (en el noreste de Brasil), se divide en dos ramas: 1) Corriente de Brasil, que se dirige hacia el sureste; y 2) Corriente de Guayana, que sigue hacia el noroeste y que, luego de unirse sus aguas a las de la Corriente Ecuatorial del norte, recurva en el Golfo de México y se dirige a Europa como Corriente del Golfo. La acción de esta doble corriente ecuatorial se manifiesta, para la Hispaniola y las demás islas del área, en una doble corriente regional.

La llamada Corriente de las Antillas baña la costa norte (Atlántica) de las Antillas Mayores y está integrada todo el año por aguas proporcionadas exclusivamente por la Corriente Ecuatorial del norte. La Corriente del Caribe baña la costa sur de las Antillas Mayores, distinguiéndose dos épocas: a) junio a noviembre -sus aguas provienen básicamente de la Corriente Ecuatorial del norte, a partir de la línea Martinica-Honduras-; y b) noviembre a junio (La Corriente Ecuatorial del sur invade totalmente el mar Caribe, desplazando a 
la Corriente Ecuatorial del norte). Hay dos tipos de corrientes en el océano: las corrientes superficiales, que constituyen el 10\% del agua del océano y se encuentran desde los $400 \mathrm{~m}$ hacia arriba, y las corrientes de agua profunda o la circulación termohalina, que afectan el otro $90 \%$ del océano.

Se establece una circulación aproximadamente constante de grandes masas de agua debido a la combinación de dos tipos de corrientes: una por la compensación de densidad y la otra por corrientes de arrastre. De este tipo de corriente, las más conocidas son las corrientes del golfo, que fluye en dirección NE y transporta las cálidas aguas del Golfo de México hasta las costas de Europa, y la corriente del labrador, que fluye en dirección contraria, desde las costas del labrador y Terra-nova, transportando agua de las regiones cálidas.

\section{Objetivo del proyecto}

El objetivo básico del proyecto es establecer un modelo de plataforma de generación eléctrica a partir del potencial energético útil de las corrientes marinas en el canal de la Mona. Para lograrlo, se deberán desarrollar los siguientes objetivos específicos:

a) Establecer los escenarios hidrológicos en función de la profundidad y la zona de los flujos marinos mediante la caracterización del modelo energético a través de las velocidades, el caudal y las direcciones de estos, para hacer el análisis previo relativo a la ubicación idónea y a la selección del material para la explotación de esos recursos.

b) Diseñar la estructura de generación electromarina más adecuada al entorno y sus posibilidades económicas-geopolíticas. Para esto se utilizarán los datos obtenidos en el apartado anterior.

c) A partir de los resultados anteriores, se desarrollará un prototipo a escala que incluya la turbina y el sistema de fijación. El sistema será ubicado en el canal para monitorizar su funcionamiento y rendimiento. 
d) Evaluar la factibilidad del manejo de los recursos energéticos en el litoral dominicano y de la implementación del modelo energético propuesto de manera práctica y provechosa al país.

\section{Problemática de investigación}

La República Dominicana enfrenta una situación de difícil solución en cuanto a su déficit energético a corto, mediano y largo plazo, donde la demanda crece a un ritmo que las nuevas plantas bajo proyecto de instalación no serán suficientes. Hasta las plantas de Punta Catalina en un par de años quedarían absorbidas y la demanda no sería satisfecha. Nuestra matriz energética depende de derivados del petróleo caros y contaminantes, así como de un insuficiente y obsoleto parque de generación, situándonos en una espiral sin fin.

Por otro lado, el canal de la Mona, ubicado en la zona contigua de las aguas litorales dominicanas, siendo el lugar de comunicación natural entre el mar Caribe y el Océano Atlántico, limitado por el cabo engaño en el este y la bahía de Mayagüez en el oeste, posee inmensas cantidades de recursos energéticos renovables no explotados. Tiene una anchura variable de $107 \mathrm{~km}$. El eje del canal transcurre de este a oeste, con una distancia de $106 \mathrm{~km}$. Sobre el paralelo $18^{\circ}$ Norte, $68^{\circ}$ Oeste. El canal posee corrientes que pueden alcanzar las 3 millas náuticas/hora $(1.54 \mathrm{~m} / \mathrm{s})$.

El potencial energético no utilizado de las corrientes marinas de nuestro litoral dominicano es inmenso al estar alimentado por las corrientes del Océano Atlántico, con su corriente del Golfo. Los investigadores estiman que esta corriente del Golfo transporta al norte unos 1,300 millones de megavatios, el equivalente a la producción de un millón de centrales energéticas. Las aguas territoriales de la República Dominicana (o, simplemente, mar dominicano) comprendidas entre sus costas, se extienden a una trazada de 6 millas náuticas (11 kilómetros) y unos $5 \mathrm{~km}$ de excelentes condiciones para la explotación energética marina en las mismas donde la profundidad está entre 200 metros 
y de 350 metros. El Estado dominicano tiene facultades exclusivas de propiedad y jurisdicción sobre ellas, así como sobre su suelo y subsuelo.

La zona contigua dominicana a una franja de otras 6 millas náuticas a partir de nuestras aguas territoriales sobre las que el país tiene derecho de patrullaje a fin de prevenir la violación de los reglamentos de aduanas, sanidad, inmigración o fiscalía, dentro de su territorio o mar territorial, pero no tiene derecho de impedir el paso de buques de otros países por ella.

Dado nuestro perenne déficit energético nacional, la pertinencia del proyecto subyace en la relevancia de nuestro aporte para desarrollar un modelo mareomotriz para la explotación energética de las corrientes oceánicas del canal de la Mona, donde tenemos unos $5 \mathrm{~km}$ de excelente planicie marina, con una batimetría adecuada para el emplazamiento de un parque mareomotriz de una gran capacidad en megavatios (1,400 MW) (que supera a cualquiera de los hasta ahora construidos en tierra firme), sostenible, cercano a nuestras costas y capaz de mitigar nuestra demanda energética insatisfecha, sin emisiones de carbono a la atmósfera y sin importación de combustibles fósiles.

\section{Métodos y materiales}

General Electric ofrece soluciones completas que trabajan para mejorar la economía de granja marea basándose en más de 10 años de experiencia acumulada. Esto incluye la exitosa prueba de $500 \mathrm{~kW}$ y 1 MW turbinas ha dado lugar a más de 1,5 GWh de electricidad se agrega a la red y ha dado datos inestimables para la industria. Oceade 1.4-18 es la primera variante de nuestra plataforma de turbinas de marea.

Con la posibilidad de ser optimizado para diferentes condiciones de sitio, nuestra plataforma de turbina mareomotriz asegura una mayor eficiencia e industrialización, así como racionalizar la operación y 
el mantenimiento. Ampliar el 1,4 MW 3-la máquina de hoja con un diámetro de rotor de $18 \mathrm{~m}$, el Oceade 1.4-18, tiene un diámetro de rotor escalable hasta a $23 \mathrm{~m}$, lo que permite seleccionar el tamaño para adaptarse a las condiciones específicas de su sitio. Como proveedor de soluciones, General Electric se enfoca en el rendimiento de todo el sistema para satisfacer las necesidades de los clientes y mejorar la economía de granja marea.

\section{Características del producto}

- Diseñada para industrialización, optimizado y mantenimiento.

- Integra la conversión de energía en la carcasa para facilitar la conexión de varias turbinas en un solo cable, eliminando la necesidad de contención adicional submarinos para electrónica de potencia.

- Posee una desviación barquilla, basada en tecnología probada para enfrentar la marea que se aproxima y maximizar la extracción de energía, minimizando cargas estructurales y efectos de Estela.

- Tiene cuchillas para control de carga en la turbina y para optimizar la respuesta a condiciones locales de la marea.

- Cuenta con portezuela trasera para mayor mantenibilidad.

- Posee carcasa flotante, permitiendo un fácil intercambio de turbina de repuesto y maximizar la disponibilidad.

\section{Modelo recomendado para el estudio energético marino con turbina LTT}

El LTT es una turbina de eje horizontal bidireccional en un tubo de venturi simétrica. El venturi dibuja las corrientes marinas existentes en el LTT para captar y convertir la energía en electricidad. El uso de un cimiento de la gravedad sumergible permitirá al LTT desplegarse rápidamente y con poca o ninguna preparación de los fondos marinos a profundidades superiores a 40 metros. Esto proporciona al LTT una clara ventaja sobre la mayoría de sus competidores y abre una fuente de energía potencial 5 veces más grande, disponible para las empresas que utilizan otras técnicas de instalación. 
El diseño y la fabricación del conducto y su estructura se basa en la tecnología y la experiencia en la industria, fabricación e instalación de petróleo y gas offshore. La nueva fundación de la LTT es carga $\mathrm{y}$ autosuficiente, sin necesidad de preparaciones de fondo marino extenso, lo que permite un proceso de rápida instalación.

Una vez en posición, el rotor de conductos bidireccional, con sus álabes simétricos, utiliza el venturi en forma de conducto para acelerar flujos de marea a través de la turbina y, por lo tanto, aumenta la energía que puede ser capturada por la turbina. El diseño del LTT y el uso de un tubo de venturi elimina la necesidad de un mecanismo de desviación a la vuelta de cada marea para mantener el dispositivo en el flujo de las mareas. El venturi en forma de conducto endereza el flujo de las mareas cuando se acerca a las láminas de turbina, garantizando el óptimo ángulo de enfoque, así como la extracción de potencia máxima de la energía disponible en el flujo. También, elimina la necesidad de control de echada de lámina; además, hay ahorros de costes significativos en diseño, construcción y mantenimiento. En el corazón de la estrategia de la energía lunar ha sido el control de los costos de O \& M. En un ambiente marino áspero, el uso de mecanismos menos complicados reduce los riesgos técnicos y los gastos de la rectificación. Un cable de alimentación convencional transmite la electricidad a la costa.

\section{Proceso conceptual del diseño}

Para seleccionar la zona de la matriz mareomotriz se cruzará la información procedente del fondo marino con la obtenida del modelo de corrientes. El objetivo es obtener distintas zonas candidatas a ubicar el parque marino. En la selección se considerarán factores importantes como el espacio y tamaño necesarios para poder ubicar un número suficiente de turbinas generadoras, la distancia y el coste de la distribución de energía (de la producción al consumidor) y las características del fondo marino (profundidad/oleaje). 
A partir de los trabajos desarrollados por UNAPEC, se propone una matriz mareomotriz con unas 140 turbinas de unos $10 \mathrm{MW}$ por unidad del Modelo Generador Tidal Stream, o unas 1,400 unidades de 1 MW (teóricamente). Así, por ejemplo, las turbinas de $1 \mathrm{MW}$ poseen hélices entre los 15 y $20 \mathrm{~m}$ de diámetro y se encuentran acopladas directamente a un engranaje multiplicador que mueve el generador eléctrico, con una potencia unitaria estimada de 500 a 1,000 KW (1 MW). Se pueden agrupar en unos 72 conjuntos de 20 unidades, de $1 \mathrm{MW}$ cada una. Así, unas 1,400 unidades de 1 MW producirían un total de 1,400 MW. Para esto se estima una longitud de unos $5 \mathrm{~km}$ donde ubicar el conjunto. Las posibles separaciones entre generadores serían de unos 60 metros (3 veces el diámetro), lo que nos daría una matriz de 12 columnas y 6 filas de profundidad, con separaciones de 100 metros (5 veces el diámetro) entre generadores, a lo ancho, para unos 2,400 metros. El rectángulo resultante de la matriz sería de unos 5,000 m x 2,400 m, para un área total del enclave de 12,000,000 de metros cuadrados, lo que equivaldría a $12 \mathrm{~km}^{2}$ (tal y como si fuera un emplazamiento eólico).

El modelaje, tanto de corrientes como de oleaje, permitirá desarrollar modelos a partir de los datos obtenidos en los objetivos anteriores. Utilizando dichos modelos se introducirán distintos modelos de turbinas para estudiar la resistencia de fluidos a que serán sometidas las turbinas, así como la energía disipada; la producción de energía también será estimada. Los sistemas de fijación de las turbinas serán modelados y sus esfuerzos estimados en función de los modelos de flujo de corriente y oleaje obtenidos. El objetivo final es el dimensionamiento adecuado de los elementos básicos de la matriz mareomotriz, por lo que hace referencia a las turbinas y sus elementos de fijación al fondo. También se modelarán las zonas muertas producidas por una turbina sobre otra turbina para así planificar una ubicación correcta de los múltiples generadores.

A partir de los datos obtenidos en objetivos anteriores, se dispone de un mapa con los puntos con mayor idoneidad para ubicar el parque 
mareomotriz, lo cual permite el desarrollo de la infraestructura necesaria para el transporte de la energía producida. Tecnológicamente, el objetivo consiste en desarrollar un estudio de la línea de transporte de energía que vaya desde la producción ubicada en el interior del mar hasta el lugar de consumo en la República Dominicana. Dicha problemática es similar a la producida en las plantas de producción eólica marinas. La solución propuesta consiste en la conversión de la corriente eléctrica en alterna producida a corriente eléctrica continua. Una vez agrupada la energía producida por los distintos generadores, una plataforma marina convierte la energía producida a corriente eléctrica continua de alto voltaje HVDC, para una transmisión más efectiva y con menos pérdidas. Una vez en la costa, una estación transformadora convierte la corriente eléctrica continua de alto voltaje a corriente alterna trifásica y se inyecta la energía a la red.

\section{Conclusión}

En esta nota técnica se discutieron los lineamientos necesarios para desarrollar un proyecto para estudiar la utilización de las corrientes marinas como fuente de generación de energía eléctrica. Se requiere de un estudio de campo para conceptualizar y modelar la plataforma energética a ser instalada en un entorno marítimo dentro de un área del canal de la Mona en estudio, el cual corresponde a la zona marítima delimitada entre la Latitud de Longitud, contando desde las costas en Punta Cana (República Dominicana), con unos 5.3 kilómetros como límite máximo del posible emplazamiento marino-energético dominicano.

Concluimos que las corrientes marinas del canal de la Mona podrían abastecer de forma sostenible y sustentable, continua, eficiente y económica unos 1,400 Mwatts al sistema energético de la República Dominicana, lo que representa el $72.5 \%$ de la demanda energética del Sistema Nacional Interconectado (SENI), de los 3,394.1 Mwatts que este maneja. Esta investigación se enmarca en "La iniciativa Energía 
Sostenible para Todos", de la cual la República Dominicana forma parte, lanzada por el Secretario General de las Naciones Unidas en el año 2011, que aspira a identificar y apoyar acciones para aumentar el estudio de la importancia de abordar las fuentes energéticas que abastecen las sociedades modernas.

\section{Bibliografía}

Camilo Reynoso, W. (2015). Estudio de factibilidad para un modelo energético sostenible a través del aprovechamiento de las corrientes marinas del canal de la Mona. Recuperado de https:// www.researchgate.net/publication/280733655.

Camilo, W., Sánchez, F., Sánchez, E., Encarnación, E., Rivera, E. \& Rivera, Y. (2015). Nonconventional electrical energy matrix using photovoltaic, solar paddles, wind mills and biogas generators. PAPER ID: EEE-1104-015, CIDTEC - Unapec.

Camilo, W. (2017). Estudio de factibilidad para un modelo energético sostenible a través del aprovechamiento de las corrientes marinas del Canal de la Mona. Santo Domingo: UNAPEC.

Dohan, K. \& Maximenko, N. (2016). Monitoring Ocean Currents with Satellite Sensors. Journal of Oceonography Society, 23(4).

Figueredo, M. (2015). Estudio de Tecnologías Energéticas Renovables. Recuperado de http://www.cubasolar.cu/biblioteca/Energia/Energia41/HTML/Articulo03.htm

Georgia Tech Research Corporation. (2016). Assessment of Energy Production Potential from Ocean Currents along the United States Coastline. Final Project Report, September 15, 2016.

Lynne, D. (2014). Descriptive Physical Oceanography: An Introduction. Academic No.184 Press., p. 254. 
Thomas, T. \& Dwarakish, G. (2015). Numerical Wave Modelling-A Review. International conference on water resources, coastal and ocean engineering (ICWRCOE'15), Vol. 4, 443-448.

Worldwatch Institute. (2015). Harnessing the Dominican Republic's Sustainable Energy Resources. Roadmap to a sustanaible energy system. Washington, D.C. 